\title{
FIBROMATOSIS IN MAXILLOFACIAL REGION
}

\author{
Jawad Ahmad Kundi ${ }^{1}$, Amina Gul Mannan', Faryal Gul' ${ }^{1}$, Mohammad Umar ${ }^{1}$ and Ajmal Khan ${ }^{1}$
}

\author{
1. Sardar Begum Dental College
}

\section{SUMMARY}

Fibromatosis is a rare and locally aggressive benign tumor arising from musculoaponeurotic structures. They are aggressive and tend to recur. Here we present a recurrent case of fibromatosis in a nineteen year old female focusing attention on the etiology, clinical presentation, pathology, various treatment options and modes of prevention of recurrence.

\section{Keywords:}

Fibromatosis, maxillofacial region

\section{INTRODUCTION}

Fibromatosis represents a group of locally aggressive benign neoplasm that shows infiltrative, destructive and recurrent growth, there is no tendency to metastasize ${ }^{1}$. They are considered to be "border line malignant and are categorized as "Grey Zone" between benign and malignant tumor ${ }^{1}$. Fibromatosis usually develop in children (hence the name Juvenile aggressive fibromatosis) and young adults (usually before age 40) ${ }^{1}$. Females are affected twice than males ${ }^{2}$. Fibromatosis is classified into two broad categories; superficial (palmer, plantar)-these do not occur in head and neck area, \& deep (dermoids), which are usually fast growing lesion and reach a significant size depending upon site of development ${ }^{1,2}$.

Deep (desmoids) are further classified anatomically as abdominal wall or intra abdominal and extra abdominal, which usually occurs in head \& neck region. They present as variously sized masses, are deep seated and there is fibrous proliferation. They usually involve soft tissue but can infiltrate the surrounding bone ${ }^{2}$. They can be sporadic, familial adenomatous polyposis associated and multicentric ${ }^{1}$ A rare case of fibromatosis in maxillofacial region is reported as under.

The purpose of this case presentation is to focus attention on this rare disorder and discuss the etiology, clinical presentation, pathology and various treatment options.

\section{CASE REPORT}

A 19 years old female came with the complaint of swelling at right side of the face and complete closure of the mouth.

\section{HISTORY OF PRESENTING ILLNESS}

According to the patient, she extracted her upper right first and second molars 5 years back, immediately after extraction, a swelling started developing on the right side of the face, this swelling was accompanied with pain. The pain increased in intensity while chewing and analgesics used to

Correspondence:

Dr. Jawad Ahmad Kundi

Sardar Begum Dental College Contact:0333-9126606

Email:drjkundi@yahoo.com https://doi.org/10.37762/igmds.2-1.62 help relieve pain. As the swelling increased the patient developed limited mouth opening. The patient got operated for this mass around 2 years back. Soon after the surgery the swelling started developing again and around 1 year back as the swelling increased in size, there was complete closure of mouth. Patient reported to us with no opening for the last one year. 


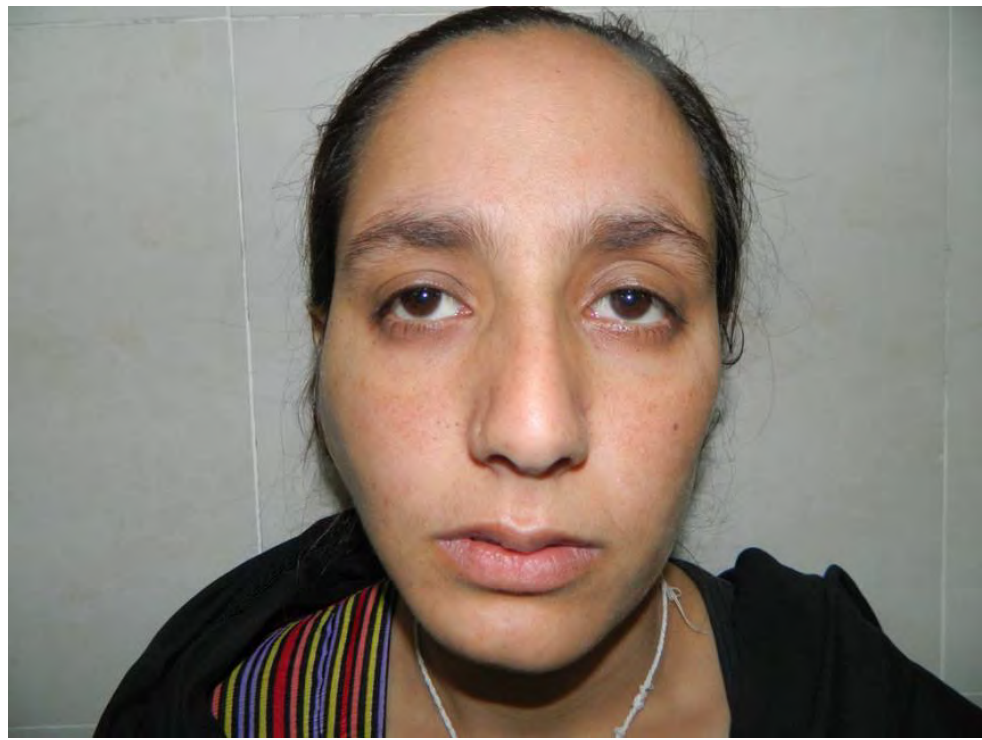

Figure 1: Pre operative image.

\section{CLINICAL FINDINGS}

- Extra oral: - Swelling on the right side of face, non tender, firm and fixed Overlying skin was normal with no color change. Facial deformity was present on right side with no lymphadenopathy. Buccal branch of facial nerve was affected in previous surgery as shown in figure 2. All other branches of facial nerve were found intact.

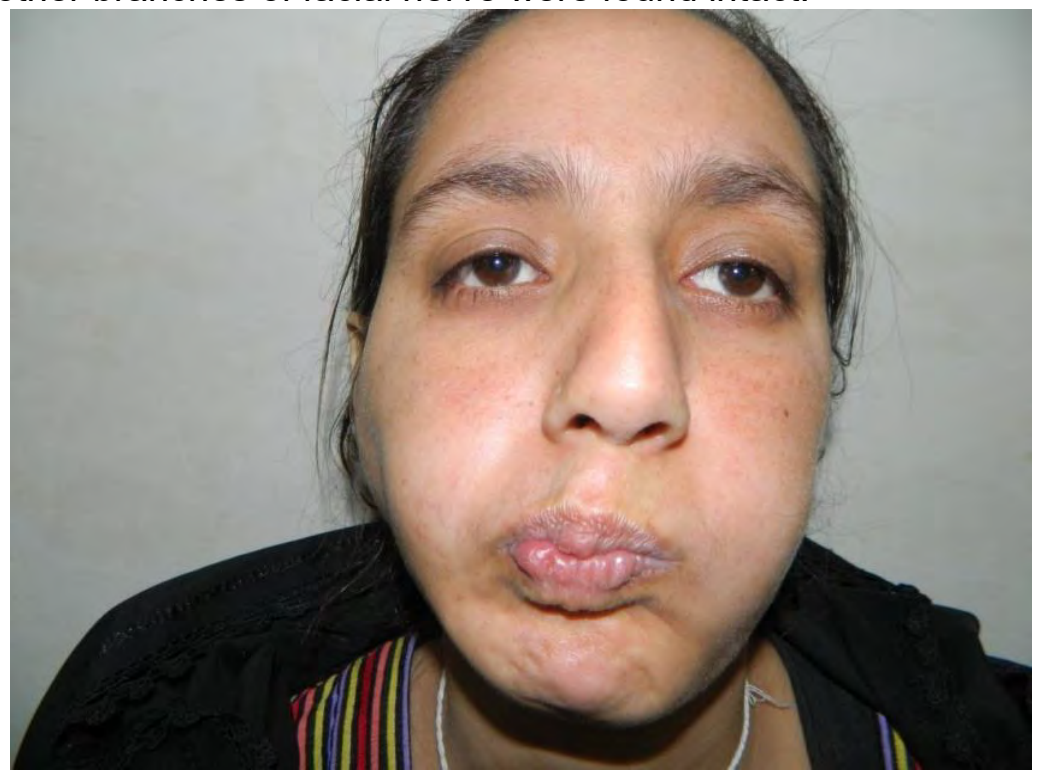

Figure 2: Affected buccal branch due to previous surgery.

- Intra oral: - Complete closure of mouth (trismus). Normal occlusion no displaced or mobile teeth, oral hygiene compromised slightly. 


\section{RADIOGRAPHIC FINDINGS}

CT scan revealed destruction of bone (buccal cortex) from the lower border of the mandible extending up to zygomatic arch (figure 3). The lesion did not invade into the adjacent bones. Axial slices revealed focal thickening of masseter muscle en-masse with partial thickening of medial pterygoid muscle.

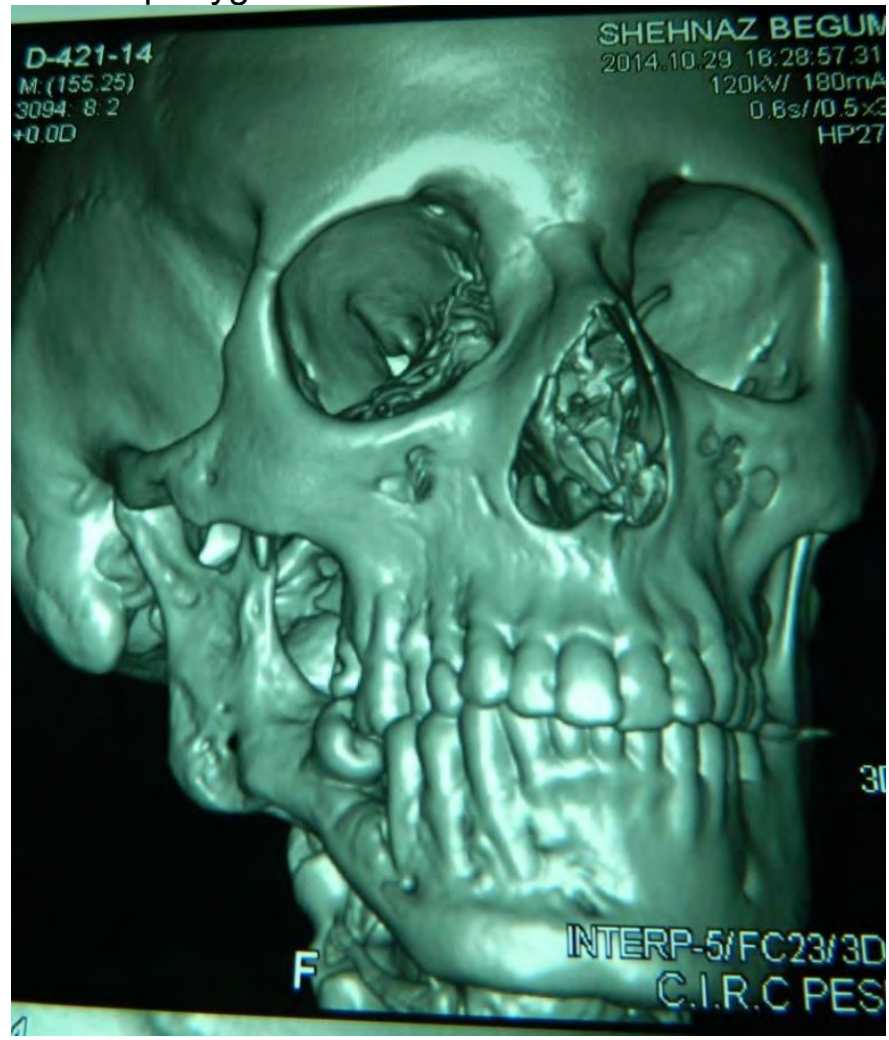

Figure: 3. CT scan image showing Destruction of bone from angle of mandible to zygomatic arch.

\section{INCISIONAL BIOPSY}

Previous biopsy revealed a proliferative process with infiltrative growth, the mass comprised spindle like elongated cells with fibroblasts/myofibroblasts. Nuclei were ovoid and uniform. Single mitotic figures were visible but no atypical mitosis were present. These cells formed interweaving large and long bundles. Spindle like cells express Vimentin, smooth muscle actin, beta Catenin, Desmin, Cytokeratin 34bE12 \& Estrogen receptor, Ki67 in approximately $5 \%$ of the nuclei

\section{PATHO MORPHOLOGICAL DIAGNOSIS}

Oral fibromatosis

\section{MANAGEMENT}

The scar from previous surgery was extended to access the lesion as shown in figure $4 a \& 4 b$. A submandibular incision was thus given \& a subplatysmal flap developed. Care was taken to raise the branches of facial nerve in the flap. Masseter muscle was dissected $\&$ stripped from ramus \& removed at its insertion. As shown in figure 5 . Superiorly it was traced upto the sigmoid notch. A further superior approach was undertaken through a Bramley-alkhayat incision \& masseter origin at zygomatic arch was stripped off. This whole 
muscle was removed piecemeal. Mouth opening of $27 \mathrm{~mm}$ (forced) \& 35mm (passive) was achieved per op. Wound was closed after hemostasis. Post op first day and fifth day facial nerve branches were found to be intact. On fifth post op day stick exercises were started \& maximal forced opening of $35 \mathrm{~mm}$ was achieved. Upon 1 month follow up CN-VII branches were found to be intact \& mouth opening still the same.

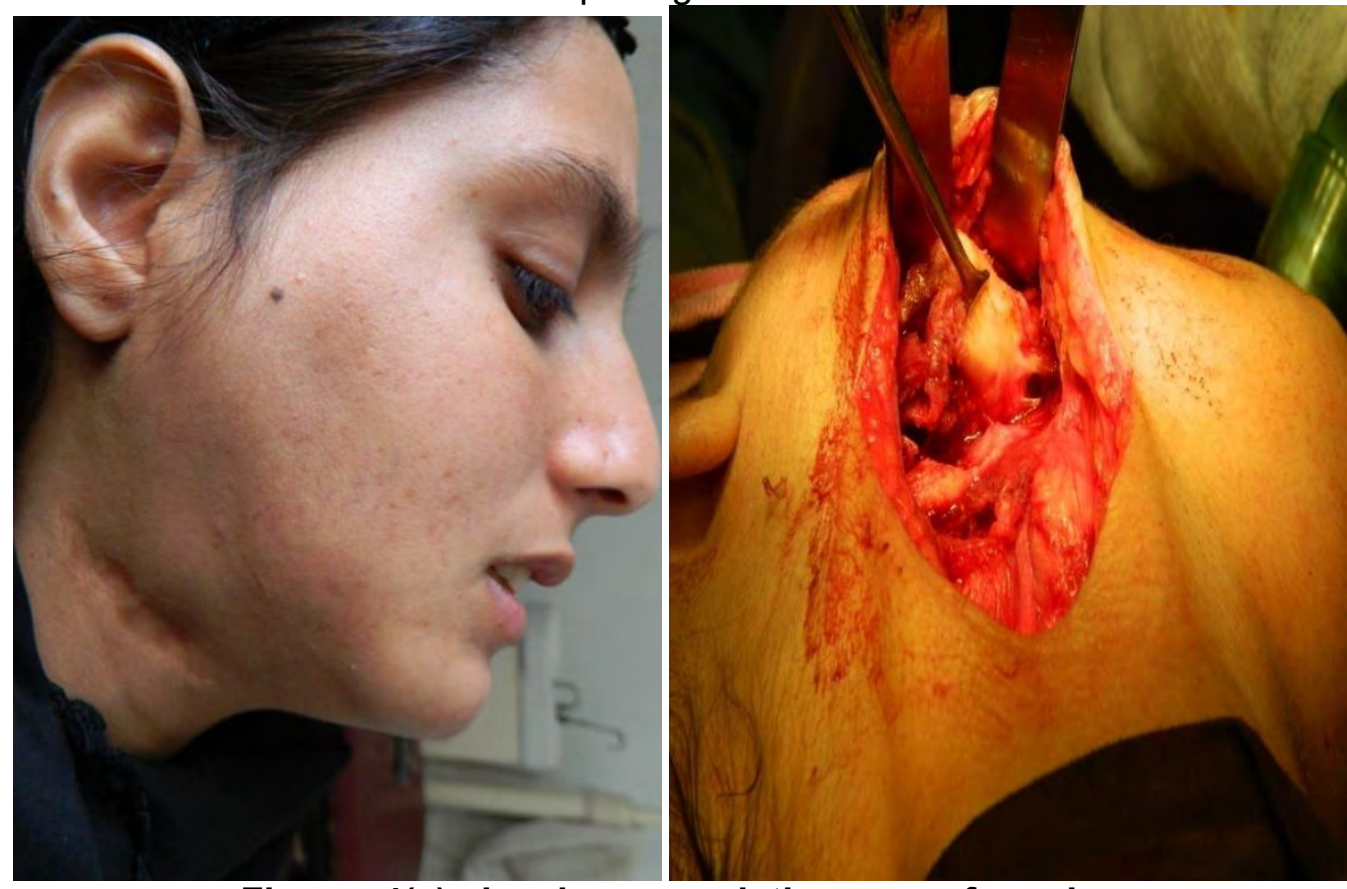

Figure: 4(a).showing pre existing scar of previous surgery, Figure: 4(b) per operative image showing the mass.

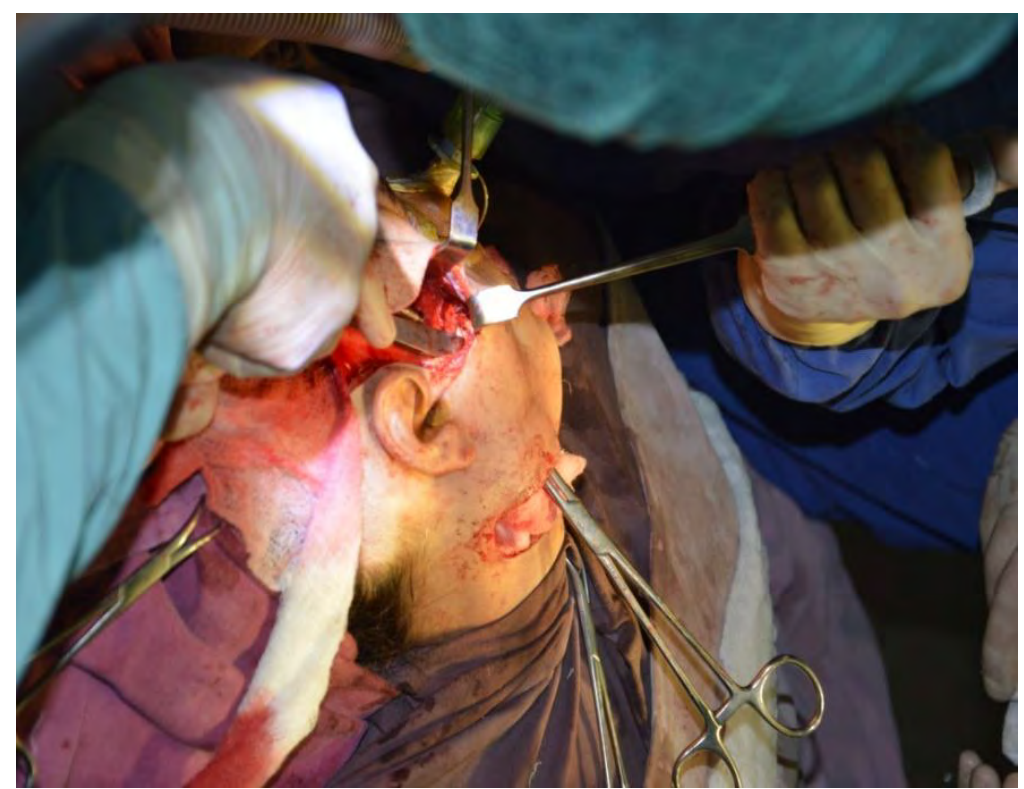

Figure: 5. showing stripping of masseter muscle 


\section{DISCUSSION}

Fibromatosis is a rare benign neoplasm which arises from musculoaponeurotic structures ${ }^{2}$. Fibrous tissue proliferation occurs with intermediate biologic behavior between a benign fibroma and fibrosarcomai.e like fibrosarcoma, it exhibits destructive, infiltrative growth that recur frequently and like fibromas, it does not metastatize. Variety of synonyms exist for fibromatosis which includes "extra articular desmoids", "desmoid tumors", 'Grade I fibrosarcoma', 'non metastatizing fibrosarcoma' and "Aggressive fibromatosis"2,1. Fibromatosis is rarely found in head \& neck region ${ }^{2,3}$. Masson and Soute reported $12 \%$ of 284 cases from all locations of body in their study while Das Gupta et all reported $11.1 \%$ of 72 cases of extra abdominal fibromatosis ${ }^{2}$. Most common site affected in the head \& neck region is supra clavicular fossa $(40-85 \%)$, which is followed by face $(25 \%)$. Other sites that are affected by fibromatosis are mandible, naso pharyngeal tract, larynx, tongue and orbit, all para nasal sinuses, infra temporal space, and para pharyngeal space ${ }^{2,1}$. The oral and maxillofacial lesions (maxilla, mandible and mastoid area) occur usually at a younger age (5 to 20 years compared to 35 years), are more infiltrative and destructive with more ability to invade the adjacent bone ${ }^{2}$. Fibromatosis is found to be more common in females, with female to male ratio $3: 2$ or 2:1. The exact etiology of fibromatosis is unknown, however trauma, such as previous surgery or blunt force injury can lead to the development of fibromatosis, the immature fibroblasts engaged in healing begin un-controlled proliferation that leads to tumor formation ${ }^{4,3,2}$. However, there have not been enough patients with head \& neck fibromatosis after local trauma to confirm this theory ${ }^{3,2}$. Endocrine factors may play a part in formation of fibromatosis because some lesions have been found to grow during puberty or pregnancy and then regress following menopause or radiation induced castration ${ }^{3,2,1}$. Some tumors have shown low level estrogen receptors. Genetic pattern in appropriate gene expression and chromosomal abnormalities have been reported in some patients with fibromatosis ${ }^{3,2}$.

For head \& neck region, fibromatosis has a high recurrence rate (20 - $70 \%$ of cases),the cause of which is unclear but it may be due to aggressive nature of tumour or due to more difficult excision of lesion ${ }^{2,4,6}$. Recurrence can occur within 2 years till up to 11 years. Fibromatosis almost never or in very few cases turn into fibrosarcoma ${ }^{2}$. The fibromatosis usually presents as slow growing, asymptomatic swelling, the mass is usually fixed to the underlying muscles, non tender, over lying skin appears normal without any color change. It often results in trismus ${ }^{3,4,5}$. Other clinical features may include nasal obstruction, epistaxis, facial pain, dyspnea, strider and proptosis depending on anatomic site of lesion. Pain and parasthesia is uncommon. Our patient came with complaint of trismus since past 1 year and swelling on right side of face. She had no complaint of pain or parasthesia ${ }^{2,3}$. In general, the clinical features were similar to other reported cases. Fibromatoisis is grayish white, firm or rubbery in consistency. Its long axis is usually oriented in direction of muscle bundles in which it is formed. It's not encapsulated and is ill defined due to its tendency to infiltrate surrounding structures. It can surround or encase vascular and neural structures without apparent invasion which is the reason of lack of pain ${ }^{1,2}$. Radiographs frequently show poorly demarcated, irregular bone destruction of lesion located at the surface of jaws \& it may show irregular resorption of adjacent cortex ${ }^{3}$. The extension of lesion, affinity for muscles, infiltrative capabilities, and clinical presentation of trismus suggested us about presence of an aggressive lesion. There was no pain or parasthesia; no lymph node enlargement \& no evidence of metastasis $^{1,2,3}$. The main treatment modality is wide excision of the lesion along with normal/ healthy margin approx. 1-1.5 cm. in head and neck region. Preservation of vital structures and their functions may impede the objective of the treatment therefore multiple modality treatment strategies are usually applied which includes surgery along with radiotherapy or chemotherapy. Anti estrogen (tamoxifen) therapy and NSAIDS (sulindar) are being used but efficacy is not established. 
Radiotherapy (50 to $60 \mathrm{GY}$ ) with surgery gives long term control. NSAIDs act probably by inhibiting prostaglandin synthesis which impairs the proliferative ability of tumor cells and at the same time stimulates immunologic response ${ }^{1,2}$. Anti estrogen (Tamoxifen) therapy is reported to regulate the speed of growth of fibromatosis especially by female sex hormones, but no definative results have yet been established. Anti cancer chemotherapy is reported to partially or completely regress fibromatosis ${ }^{1,2,3}$.

\section{CONCLUSION}

Fibromatosis is a benign, locally aggressive neoplasm. It is rarely found in oral and maxillofacial region. In most cases, the lesion is usually asymptomatic and patient seeks treatment either when the lesion grows considerable in size and results in facial deformity or when lesion involves surrounding structures and results in trismus. Surgery is the treatment of choice with 1-1.5 $\mathrm{cm}$ healthy margins. Long term follow up is necessary due to the high reccurence rate of the disease.

\section{REFERENCES}

1. GeorgiP.Ivanov,Dimitar T. Atanasov, BeniaminL.Anavi.Aggnessive juvenile mandibular fibromatosis. foliaMedica. 2013 Nov; 55(3\&4):90-93

2. Ariful Islam, MahfuzHossain, FazlaRubbyTymur,AlauddinAlazad, TarifuzzamanRubel. Fibromatosis of the maxillary sinus and muscles of mastication: a case report. UpDatDen. Coll. J 2013; 3(2): 4854

3. Ying-Nan Chang, Yuan - Chien Chuang, Yaoh-Shiang Lin. Fibromatosis of the submandibular region. $J$ chin Med Assoc. 2009 Sep; 72(9): 492-494

4. Joseph A. Regezi, James J. Sciubba. Oral pathology Clinical pathological correlations. 6th ed.St.Louis: Saunders Elsevier; 2012

5. Sze H. Yeung MW. Fibromatosis of the neck causing airway obstruction managed effectively with weekly low-dos methotrexate and vinblastine. Hong Kong Med J2009; 15:221-223.

6. Sobani ZA, Junaid M, Khan MJ.Successful management of aggressive fibromatosis of the neck using wide surgical excision: a case report. J med case reports 2011; 5:244

7. Kruse AL. Luebbers HT. Gratz KW. Obwegeser JA. Aggressive fibromatosis of the head and neck: a new classification based on a literature review over 40 years (1968-2008). Oral MaxillofacSurg 2010; 14(4): 227-232.

8. Sharma A. Nagan BY.Sandor GK. Sandor GK.Campisi P. Forte V. Pediatrid aggressive fibromatosis of the head and neck: a 20-years retrospective review. J PediatrSueg 2008; 43: 1596-1604.

9. Chiara Y. Mochiki M. Sagasawa M. Nibu K. Fibromatosis of the infratemporal fossa extending to rheparapharyngealspaceaccopanied by trismus. AurNasLar, 2003; 30: 319 - 323.

10. Marx re. Stern D. Oral and Macillofacial Pathology; A Rationale for Diagnosis and Treatment. Quintessence Publishing Co. Inc. P 463 - 465.

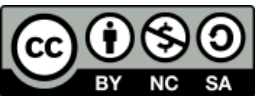

$$
\begin{aligned}
& \text { LICENSE: IGMDS publishes its articles under a Creative Commons Attribution Non-Commercial Share-Alike license (CC-BY-NC-SA 4.0). } \\
& \text { COPYRIGHTS: Authors retain the rights without any restrictions to freely download, print, share and disseminate the article for any lawful }
\end{aligned}
$$

